\title{
One dimensional fractional frequency Sumudu transform by inverse $\alpha$-difference operator
}

B. Chandrasekar ${ }^{*}$, M. Meganathan ${ }^{2}$ and S. Vasuki ${ }^{3}$

1,2,3 Department of Mathematics, Sacred Heart College (Autonomous),

Tirupattur - 635601, Tirupattur District

Tamil Nadu, S.India.

\begin{abstract}
In this paper, we define fractional frequency Sumudu transform by inverse $\alpha$-difference operator. Here we present certain new results on Sumudu transform of polynomial factorial, trigonometric and geometric functions using shift value. Finally, we provide the relation between convolution product and fractional Sumudu transform of polynomial and exponential function. Numerical results are verified and analysed the outcomes by graphs.

Key words: $\alpha(h)$-difference operator, fractional difference, extorial function, gamma function and polynomial factorial.
\end{abstract}

AMS classification:47B39, 39A70, 11J54, 33B15

\section{Introduction}

There are several integral transforms such as the Laplace, Millen, Hankel and Fourier transforms that are used to solve differential equations which apper in many fields of science and engineering. In the early 1990's, Watugala [9, 10] introduced the Sumudu transform and applied it to solve ordinary differential equations. Watugala's work was followed by Weerakoon who introduced the complex inversion formula for the Sumudu transform [11, 12]. The fundamental properties of this transform, which is thought to be an alternative to the Laplace transform were then established in many articles [13, 14].

\footnotetext{
${ }^{1 *}$ chandrasekarmaths81@gmail.com, ${ }^{2}$ meganathanmath@gmail.com, ${ }^{3}$ vasukishc@gmalilagøend 37 of 152
} 
The Sumudu transform is defined over the set of functions

$$
A=\left\{f(t)\left|\exists M, \tau_{1}, \tau_{2}>0,\right| f(t) \mid<M e^{\frac{|t|}{\tau_{j}}}, i f t \in(-1)^{j} \times[0, \infty)\right\},
$$

by

$$
F(u)=\mathbb{S}\{f(t)\}(u)=\frac{1}{u} \int_{0}^{\infty} f(t) e^{-\frac{t}{u}} d t, u \in\left(-\tau_{1}, \tau_{2}\right) .
$$

Also, there is an bridge between Laplace transform and Sumudu transform which has many applications in applied sciences. Moreover, some properties of Sumudu transform makes it more advantageous than the Sumudu transform of a Heaviside step function is a also Heaviside step function in the transformed domain;

$$
\begin{aligned}
-\mathbb{S} t^{n} & =n ! u^{n} ; \\
-\lim _{t \rightarrow \infty} f(t) & =\lim _{u \rightarrow 0} F(u) ; \\
-\lim _{t \rightarrow 0} f(t) & =\lim _{u \rightarrow 0} F(u) ; \\
-i f c>0, \mathbb{S} f(c t) & =F(c u)
\end{aligned}
$$

Recently, it was proved that by using the Sumudu transform, one can transform the two dimensional transport equation into a Fredholm integral equation [18]. In [17], the authors applied the Sumudu transform to fractional differential equations which have many applications in the fields of science (see [19] and the references therein).

Begin with classical definition of Laplace transform an arbitrary time scales, the concept of the $h$-Laplace and consequently the discrete Laplace transformed were specified in [15]. It was initiated by Stefan Hilger [16]. This theory is a tool that unifies the theories of continuous and discrete time systems. It is the subject of recent studies on many different fields in which dynamic process can be described with discrete or continuous models. The recent applications of fractional Laplace transform using difference equation are found in [1, 2, 20, 21, 22].

In this research article, we proposed a new type of Sumudu transform with shift value and the properties are discussed. Several results are derived to validate the definition and also the relation between convolution product and sumudu transform are played a vital role using $\alpha$-difference operator.

${ }^{1 *}$ chandrasekarmaths81@gmail.com, ${ }^{2}$ meganathanmath@gmail.com, ${ }^{3}$ vasukishc@gmalliggond 38 of 152 
ISSN: 2456-8686, Volume 4, Issue 1, 2020:137-152

DOI : http://doi.org/10.26524/cm73

\section{Preliminaries}

In this section, we present basic theory of the $h$-difference operator $\Delta_{h}$. The polynomial factorial is defined $t_{h}^{(m)}=t(t-h)(t-2 h) \cdots(t-(m-1) h), h>0$ for non-negative integer $m$ and using Stirling numbers of first kind $s_{r}^{m}$ and second kind $S_{r}^{m}$, the relation between polynomial and polynomial factorials are given by,

$$
\text { (i) } t_{h}^{(m)}=\sum_{r=1}^{m} s_{r}^{m} h^{m-r} t^{r},(i i) t^{m}=\sum_{r=1}^{m} S_{r}^{m} h^{m-r} t_{h}^{(r)} \text {. }
$$

Definition 2.1 Let $u(t), t \in[0, \infty)$, be a real or complex valued function and $h>0$ be a fixed shift value. Then, the $\alpha(h)$-difference operator $\Delta_{\alpha(h)}$ on $u(t)$ is defined as

$$
\Delta_{\alpha(h)} u(t)=\frac{u(t+h)-\alpha u(t)}{h}
$$

and its infinite $h$ - difference sum is defined by

$$
\left.\Delta_{\alpha(h)}^{-1} u(t)\right|_{0} ^{\infty}=h \sum_{r=0}^{\infty} \alpha^{-(r+1)} u(t+r h)
$$

Remark 2.2 When $\alpha=h=1$ in (4) we get $\Delta u(t)=u(t+1)-u(t)$.

Lemma 2.3 Let $u(t)$ and $v(t)$ are the two real valued functions defined on $(-\infty, \infty)$ and if $\Delta_{\alpha(h)} v(t)=u(t)$, then the finite inverse principle law is given by

$$
v(t)-\alpha^{m} v(t-m h)=h \sum_{r=1}^{m} \alpha^{r-1} u(t-r h), m \in Z^{+}
$$

Proof. Let $\Delta_{\alpha(h)} v(t)=u(t)$, which gives

$\frac{v(t+h)-\alpha v(t)}{h}=u(t) \Rightarrow v(t+h)-\alpha v(t)=h u(t)$

Replace $t$ by $t-h$, we get

$$
v(t)-\alpha v(t-h)=h u(t-h) \text {. }
$$

Replace $t$ by $t-2 h, t-3 h, \ldots$ and proceeding like this in general, we get

$v(t)-\alpha^{m} v(t-m h)=h \sum_{r=1}^{m} \alpha^{r-1} u(t-r h)$.

1* chandrasekarmaths81@gmail.com, ${ }^{2}$ meganathanmath@gmail.com, ${ }^{3}$ vasukishc@gmaillag@m 39 of 152 
Lemma 2.4 [22] Let $h>0$ and $u(t), w(t)$ are real valued bounded functions. Then

$$
\Delta_{\alpha(h)}^{-1}(u(t) w(t))=u(t) \Delta_{\alpha(h)}^{-1} w(t)-\Delta_{\alpha(h)}^{-1}\left(\Delta_{\alpha(h)}^{-1} w(t+h) \Delta_{h} u(t)\right) .
$$

Lemma 2.5 Let $t \in(-\infty, \infty), h>0, \tau \in R$ and $\nu>0$, then we have

$$
\Delta_{\alpha(h)}^{-1} e^{-\frac{t}{\tau^{\nu}}}=\frac{h e^{-\frac{t}{\tau^{\nu}}}}{\left(e^{-\frac{h}{\tau^{\nu}}}-\alpha\right)}
$$

Proof. Taking $u(t)=e^{-\frac{t}{\tau^{\nu}}}$ in Definition 2.1, we have

$$
\Delta_{\alpha(h)} e^{-\frac{t}{\tau^{\nu}}}=\frac{e^{-\frac{1}{\tau^{\nu}}(t+h)}-\alpha e^{\frac{t}{\tau^{\nu}}}}{h}=\frac{e^{-\frac{t}{\tau^{\nu}}}\left[e^{-\frac{h}{\tau^{\nu}}}-\alpha\right]}{h} .
$$

Apply $\Delta_{\alpha(h)}^{-1}$ on both sides, we get (8).

Corollary 2.6 Let $t \in(-\infty, \infty), h>0 \tau \in R$ and $\nu>0$, then we have

$$
\frac{h e^{-\frac{t}{\tau^{\nu}}}}{\left(e^{-\frac{h}{\tau^{\nu}}}-\alpha\right)}-\alpha^{m} \frac{h e^{-\frac{t-m h}{\tau^{\nu}}}}{\left(e^{-\frac{h}{\tau^{\nu}}}-\alpha\right)}=h \sum_{r=1}^{m} \alpha^{r-1} e^{\frac{-(t-r h)}{\tau^{\nu}}} .
$$

Proof. The proof follows by equating (8) and using (6).

Example 2.7 For the particular values $h=4, t=3, \nu=0.2, \tau=2, \alpha=2$ and $m=3$, (9) is verified by MATLAB. The coding is given by $(4 . * \exp (-3 . / 2 . \wedge(0.2))) \cdot /(\exp (-4 . / 2 . \wedge(0.2))-2)-(32 . * \exp (9 . / 2 . \wedge(0.2))) \cdot /(\exp$ $(-4 . / 2 . \wedge(0.2))-2)=4 . * \operatorname{symsum}(2 . \wedge(r-1) . * \exp ((-3+r . * 4) . /(2 . \wedge(0.2))), r, 1,3)$.

Theorem 2.8 Let $t \in(-\infty, \infty), h>0, \tau \in R, \nu>0$ be shift value and 
ISSN: 2456-8686, Volume 4, Issue 1, 2020:137-152

DOI : http://doi.org/10.26524/cm73

$e^{-\frac{h}{\tau^{\nu}}}-2 \alpha \cos p h+\alpha^{2} e^{\frac{h}{\tau^{\nu}}} \neq 0$. Then, we have

$$
\begin{aligned}
& \Delta_{\alpha(h)}^{-1}\left(e^{-\frac{t}{\tau^{\nu}}} \cos p t\right)=\frac{h e^{\frac{h}{\tau^{\nu}}} e^{-\frac{t}{\tau^{\nu}}\left(e^{-\frac{h}{\tau^{\nu}}} \cos p(t-h)-\alpha \cos p t\right)}}{e^{-\frac{h}{\tau^{\nu}}}-2 \alpha \cos p h+\alpha^{2} e^{\frac{h}{\tau^{\nu}}}} \\
& \Delta_{\alpha(h)}^{-1}\left(e^{-\frac{t}{\tau^{\nu}}} \sin p t\right)=\frac{h e^{\frac{h}{\tau^{\nu}}} e^{-\frac{t}{\tau^{\nu}}}\left(e^{-\frac{h}{\tau^{\nu}}} \sin p(t-h)-\alpha \sin p t\right)}{e^{-\frac{h}{\tau^{\nu}}}-2 \alpha \cos p h+\alpha^{2} e^{\frac{h}{\tau^{\nu}}}}
\end{aligned}
$$

Proof. Taking $u(t)=e^{-\frac{t}{\tau^{\nu}}} v(t)=\cos p t$ in (7), we get

$$
\begin{aligned}
& \Delta_{\alpha(h)}^{-1}\left(e^{-\frac{t}{\tau^{\nu}}} \cos p t\right)=\operatorname{Re} \operatorname{part} \Delta_{\alpha(h)}^{-1}\left(e^{-\frac{t}{\tau^{\nu}}} e^{i p t}\right)=\operatorname{Re} \text { part } \Delta_{\alpha(h)}^{-1}\left(e^{\left(-\frac{1}{\tau^{\nu}}+i p\right) t}\right) . \\
& \text { Now } \Delta_{\alpha(h)} e^{\left(-\frac{1}{\tau^{\nu}}+i p\right) t}=\frac{e^{\left(-\frac{1}{\tau^{\nu}}+i p\right)(t+h)}-\alpha e^{\left(-\frac{1}{\tau^{\nu}}+i p\right) t}}{h}=\frac{e^{\left(-\frac{1}{\tau^{\nu}}+i p\right) t}\left[e^{\left(-\frac{1}{\tau^{\nu}}+i p\right) h}-\alpha\right]}{h} \text {. }
\end{aligned}
$$

Taking $\Delta_{\alpha(h)}^{-1}$ on both sides, we arrives

$$
\Delta_{\alpha(h)}^{-1} e^{\left(-\frac{1}{\tau^{\nu}}+i p\right) t}=\frac{h e^{\left(-\frac{1}{\tau^{\nu}}+i p\right) t}}{\left(e^{\left(-\frac{1}{\tau^{\nu}}+i p\right) h}-\alpha\right)}
$$

$\operatorname{Re} p a r t \Delta_{\alpha(h)}^{-1} e^{\left(-\frac{1}{\tau^{\nu}}+i p\right) t}=\operatorname{Re} \operatorname{part} \frac{h e^{\left(-\frac{1}{\tau^{\nu}}+i p\right) t}}{\left(e^{\left(-\frac{1}{\tau^{\nu}}+i p\right) h}-\alpha\right)\left(e^{\left(-\frac{1}{\tau^{\nu}}+i p\right) h}-\alpha\right)}$

$$
=\operatorname{Re} \operatorname{part} \frac{h\left[e^{-\frac{1}{\tau^{\nu}}(t+h)} e^{i p(t-h)}-\alpha e^{\left(-\frac{1}{\tau^{\nu}}+i p\right) t}\right]}{e^{-\frac{2 h}{\tau^{\nu}}}-\alpha e^{-\frac{h}{\tau^{\nu}}}\left(e^{i p h}+e^{-i p h}\right)+\alpha^{2}} .
$$

On simplifying the above expression we get (10). Similarly we get (11).

${ }^{1 *}$ chandrasekarmaths81@gmail.com, ${ }^{2}$ meganathanmath@gmail.com, ${ }^{3}$ vasukishc@gmaflag@ent 41 of 152 
ISSN: 2456-8686, Volume 4, Issue 1, 2020:137-152

DOI : http://doi.org/10.26524/cm73

\section{Alpha Fractional Frequency Sumudu Transform}

In this section, we derive several results and identities on fractional frequency Sumudu transform of polynomial factorial, trigonometric and geometric functions.

Definition 3.1 Let $u(t)$ be the real valued function, $h>0$ and $\nu \in R^{+}$. If $\lim _{t \rightarrow \infty} \Delta_{\alpha(h)}^{-1} u(t) e^{-\frac{t}{\tau^{\nu}}}=0$, then the fractional frequency Sumudu transform with tuning factor $\alpha$ is defined as

$$
{ }_{\alpha} \mathbb{S}_{h, \nu}(u(t))=\left.\frac{1}{\tau} \Delta_{\alpha(h)}^{-1} u(t) e^{-\left(\frac{t}{\tau^{\nu}}\right)}\right|_{0} ^{\infty}=-\frac{h}{\tau} \sum_{r=0}^{\infty} \alpha^{-(r+1)} u(r h) e^{-\left(\frac{r h}{\tau^{\nu}}\right)}
$$

Theorem 3.2 If $e^{-\frac{h}{\tau^{\nu}}}-2 \alpha \cos p h+\alpha^{2} e^{\frac{h}{\tau^{\nu}}} \neq 0$, then we have

$$
\begin{aligned}
{ }_{\alpha} \mathbb{S}_{h, \nu}[\sin p t] & =\frac{1}{\tau} \frac{h \sin p h}{e^{-\frac{h}{\tau^{\nu}}}-2 \alpha \cos p h+\alpha^{2} e^{\frac{h}{\tau^{\nu}}}}, \\
{ }_{\alpha} \mathbb{S}_{h, \nu}[\cos p t] & =\frac{1}{\tau} \frac{h\left(\alpha e \frac{h}{\tau^{\nu}}-\cos p h\right)}{e^{-\frac{h}{\tau^{\nu}}}-2 \alpha \cos p h+\alpha^{2} e^{\frac{h}{\tau^{\nu}}}} .
\end{aligned}
$$

Proof. The proof of (13) follows by multiplying $\frac{1}{\tau}$ and applying limits from 0 to $\infty$ in (10) and 11). The following example is the numerical verification of Theorem (3.2)

Example 3.3 For the particular values $\nu=0.5, \tau=2, p=3, \alpha=3$ and $h=3$, 13 is verifieed by $M A T L A B$. The coding is given by $(3 . *(\exp ((3) \cdot /(2 . \wedge(0.5))-$ $\cos (3 . * 3)) \cdot /(2 . * \exp (-3 . / 2 . \wedge(0.5))-6 . * \cos (9)+9 . * \exp (3 . / 2 . \wedge(0.5)))=$ $(3 . / 2) . * \operatorname{symsum}(3 . \wedge(-(r+1)) . * \cos (3 . * 3 . * r) . * \exp ((-r . * 3) . /(2 . \wedge(0.5)), r, 0$, inf $)$

Remark 3.4 When $h \rightarrow 0$ and $\nu=1$, in (3.2) we arrive $\mathbb{S}(\sin p t)=\frac{p}{\tau^{2}+p^{2}} \quad$ and $\mathbb{S}(\cos p t)=\frac{\tau}{\tau^{2}+p^{2}}$

\footnotetext{
${ }^{1 *}$ chandrasekarmaths81@gmail.com, ${ }^{2}$ meganathanmath@gmail.com, ${ }^{3}$ vasukishc@gmalliag@rd 42 of 152
} 
ISSN: 2456-8686, Volume 4, Issue 1, 2020:137-152

DOI : http://doi.org/10.26524/cm73

Theorem 3.5 If $e^{-\left(\frac{t}{\tau^{\nu}} \pm p\right) h} \neq \alpha$ and $\tau>0$, then

$$
\begin{aligned}
& { }_{\alpha} \mathbb{S}_{h, \nu}(\sinh p t)=\frac{h}{2 \tau}\left[\frac{1}{e^{-\left(\frac{1}{\tau^{\nu}}+p\right) h}-\alpha}+\frac{1}{\left.\alpha-e^{-\left(\frac{1}{\tau^{\nu}}-p\right) h}\right]},\right. \\
& { }_{\alpha} \mathbb{S}_{h, \nu}(\cosh p t)=\frac{h}{2 \tau}\left[\frac{1}{\alpha-e^{-\left(\frac{1}{\tau^{\nu}}-p\right) h}}+\frac{1}{\alpha-e^{-\left(\frac{1}{\tau^{\nu}}+p\right) h}}\right] .
\end{aligned}
$$

Proof. From the definition of Sumudu transform, we have

$$
\begin{aligned}
{ }_{\alpha} \mathbb{S}_{h, \nu}(\cosh p t) & =\left.\frac{1}{2 \tau} \Delta_{\alpha(h)}^{-1}\left(e^{p t}+e^{-p t}\right) e^{-\left(\frac{t}{\tau^{\nu}}\right)}\right|_{0} ^{\infty} \\
& =\frac{1}{2 \tau}\left[\Delta_{\alpha(h)}^{-1} e^{-\left(\frac{1}{\tau^{\nu}}-p\right) t}+\Delta_{\alpha(h)}^{-1} e^{-\left(\frac{1}{\tau^{\nu}}+p\right) t}\right] \cdot \\
\text { Now }, \Delta_{\alpha(h)} e^{-\left(\frac{1}{\tau^{\nu}}-p\right) t} & =\frac{e^{-\left(\frac{1}{\tau^{\nu}}-p\right)(t+h)}-\alpha e^{-\left(\frac{1}{\tau^{\nu}}-p\right) t}}{h}=\frac{e^{-\left(\frac{1}{\tau^{\nu}}-p\right) t}\left[e^{-\left(\frac{1}{\tau^{\nu}}-p\right) h}-\alpha\right]}{h} .
\end{aligned}
$$

Apply $\Delta_{\alpha(h)}^{-1}$ on both sides we get,

$$
\begin{aligned}
\Delta_{\alpha(h)}^{-1} e^{-\left(\frac{1}{\tau^{\nu}}-p\right) t} & =\frac{h e^{-\left(\frac{1}{\tau^{\nu}}-p\right) t}}{\left(e^{-\left(\frac{1}{\tau^{\nu}}-p\right) h}-\alpha\right)} . \\
\Delta_{\alpha(h)}^{-1} e^{-\left(\frac{1}{\tau^{\nu}}+p\right) t} & =\frac{h e^{-\left(\frac{1}{\tau^{\nu}}+p\right) t}}{\left(e^{-\left(\frac{1}{\tau^{\nu}}+p\right) h}-\alpha\right)} .
\end{aligned}
$$

${ }^{1 *}$ chandrasekarmaths81@gmail.com, ${ }^{2}$ meganathanmath@gmail.com, ${ }^{3}$ vasukishc@gmaflaggend 43 of 152 
ISSN: 2456-8686, Volume 4, Issue 1, 2020:137-152

DOI : http://doi.org/10.26524/cm73

$$
\begin{aligned}
& { }_{\alpha} \mathbb{S}_{h, \nu}(\cosh p t)=\frac{1}{2 \tau}\left[\frac{h e^{-\left(\frac{1}{\tau^{\nu}}-p\right) t}}{\left(e^{-\left(\frac{1}{\tau^{\nu}}-p\right) h}-\alpha\right)}+\frac{h e^{-\left(\frac{1}{\tau^{\nu}}+p\right) t}}{\left(e^{-\left(\frac{1}{\tau^{\nu}}+p\right) h}-\alpha\right)}\right], \\
& =\frac{h}{2 \tau}\left[-\frac{1}{\left(e^{-\left(\frac{1}{\tau^{\nu}}-p\right) h}-\alpha\right)}-\frac{1}{\left(e^{-\left(\frac{1}{\tau^{\nu}}+p\right) h}-\alpha\right)}\right] \text {, }
\end{aligned}
$$

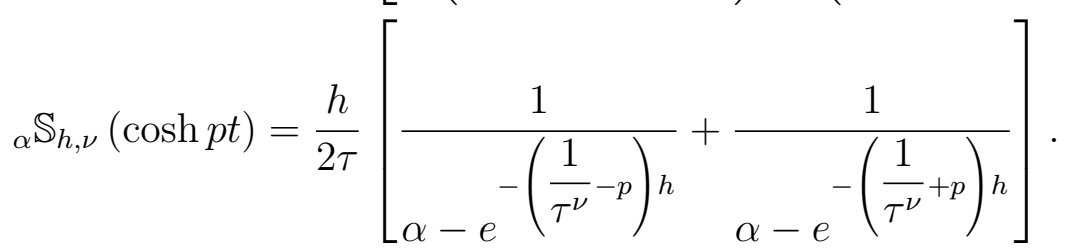

In the similar manner, we arrives

$$
\begin{aligned}
{ }_{\alpha} \mathbb{S}_{h, \nu}(\sin h p t) & \left.=\left.\frac{1}{2 \tau} \Delta_{\alpha(h)}^{-1}\left(e^{p t}-e^{-p t}\right) e^{-\left(\frac{t}{\tau^{\nu}}\right)}\right|_{0} ^{\infty}{ }^{-1} e^{-\left(\frac{1}{\tau^{\nu}}+p\right) t}\right] \\
& =\frac{1}{2 \tau}\left[\Delta_{\alpha(h)}^{-1} e^{-\left(\frac{1}{\tau^{\nu}}-p\right) t}\right] \\
{ }_{\alpha} \mathbb{S}_{h, \nu}(\sinh p t) & =\frac{1}{2 \tau}\left[\frac{h e^{-\left(\frac{1}{\tau^{\nu}}-p\right) t}}{\left(\frac{1}{\tau^{\nu}}-p\right) h}-\frac{h e^{-\left(\frac{1}{\tau^{\nu}}+p\right) t}}{\left(e^{-\left(\frac{1}{\tau^{\nu}}+p\right) h}-\alpha\right)}\right], \\
= & \frac{h}{2 \tau}\left[\frac{\left(e^{-\left(\frac{1}{\tau^{\nu}}+p\right) h}-\alpha\right)}{\left.\left(e^{-\left(\frac{1}{\tau^{\nu}}-p\right) h}-\alpha\right)\right]}\right],
\end{aligned}
$$

${ }^{1 *}$ chandrasekarmaths81@gmail.com, ${ }^{2}$ meganathanmath@gmail.com, ${ }^{3}$ vasukishc@gmaflaggend 44 of 152 
ISSN: 2456-8686, Volume 4, Issue 1, 2020:137-152

DOI : http://doi.org/10.26524/cm73

$$
{ }_{\alpha} \mathbb{S}_{h, \nu}(\sinh p t)=\frac{h}{2 \tau}\left[\frac{1}{e^{-\left(\frac{1}{\tau^{\nu}}+p\right) h}-\alpha}+\frac{1}{\left.\alpha-e^{-\left(\frac{1}{\tau^{\nu}}-p\right) h}\right]}\right.
$$

which completes the proof of $(15)$.

Remark 3.6 When $h \rightarrow 0$ and $\nu=1$, we get $\mathbb{S}(\sinh p t)=\frac{p}{\tau^{2}-p^{2}}$ and $\mathbb{S}(\cosh p t)=\frac{\tau}{\tau^{2}-p^{2}}$.

Theorem 3.7 Let $t \in(0, \infty), h>0$ and $\tau>0$, then

$$
{ }_{\alpha} \mathbb{S}_{h, \nu}\left(t_{h}^{(n)}\right)=(-1)^{n+1} \frac{h^{n+1} n ! e^{\frac{-n h}{\tau^{\nu}}}}{\tau\left(e^{\frac{-h}{\tau^{\nu}}}-\alpha\right)^{n+1}} .
$$

Proof. Taking $u(t)=t_{h}^{(1)}, w(t)=e^{-\frac{t}{\tau^{\nu}}}$ in (6), we get

$$
\begin{aligned}
\Delta_{\alpha(h)}^{-1} t_{h}^{(1)} e^{-\frac{t}{\tau^{\nu}}} & =t_{h}^{(1)} \Delta_{\alpha(h)}^{-1} e^{-\frac{t}{\tau^{\nu}}}-\Delta_{\alpha(h)}^{-1}\left[\Delta_{\alpha(h)}^{-1} e^{-\frac{1}{\tau^{\nu}}(t+h)} \Delta_{h} t_{h}^{(1)}\right] \\
& =t_{h}^{(1)} \frac{h e^{-\frac{t}{\tau^{\nu}}}}{\left(e^{-\frac{h}{\tau^{\nu}}}-\alpha\right)}-\Delta_{\alpha(h)}^{-1}\left[\frac{h e^{-\frac{1}{\tau^{\nu}}(t+h)}}{h}\right] \\
& =t_{h}^{(1)} \frac{\left.\left.h e^{-\frac{h}{\tau^{\nu}}}-\alpha\right)\right]}{\left(e^{-\frac{h}{\tau^{\nu}}}-\alpha\right)}-\frac{\left(e^{-\frac{h}{\tau^{\nu}}}-\alpha\right)}{h}\left[\Delta_{\alpha(h)}^{-1} e^{-\frac{1}{\tau^{\nu}}(t+h)}\right] \\
\Delta_{\alpha(h)}^{-1} t_{h}^{(1)} e^{-\frac{t}{\tau^{\nu}}} & =t_{h}^{(1)} \frac{h e^{-\frac{t}{\tau^{\nu}}}}{\left(e^{-\frac{h}{\tau^{\nu}}}-\alpha\right)}-\frac{h^{2} e^{-\frac{1}{\tau^{\nu}}(t+h)}}{\left(e^{-\frac{h}{\tau^{\nu}}}-\alpha\right)^{2}}
\end{aligned}
$$

${ }^{1 *}$ chandrasekarmaths81@gmail.com, ${ }^{2}$ meganathanmath@gmail.com, ${ }^{3}$ vasukishc@gmaflaggent 45 of 152 
ISSN: 2456-8686, Volume 4, Issue 1, 2020:137-152

DOI : http://doi.org/10.26524/cm73

$$
\begin{aligned}
& \left.\Delta_{\alpha(h)}^{-1} t_{h}^{(1)} e^{-\frac{t}{\tau^{\nu}}}\right|_{t=0} ^{\infty}=t_{h}^{(1)} \frac{h e^{-\frac{t}{\tau^{\nu}}}}{\left(e^{-\frac{h}{\tau^{\nu}}}-\alpha\right)}-\left.\frac{h^{2} e^{-\frac{1}{\tau^{\nu}}(t+h)}}{\left(e^{-\frac{h}{\tau^{\nu}}}-\alpha\right)^{2}}\right|_{t=0} ^{\infty} \\
& \left.\frac{1}{\tau} \Delta_{\alpha(h)}^{-1} t_{h}^{(1)} e^{-\frac{t}{\tau^{\nu}}}\right|_{t=0} ^{\infty}=\frac{h^{2} e^{-\frac{h}{\tau^{\nu}}}}{\tau\left(e^{-\frac{h}{\tau^{\nu}}}-\alpha\right)^{2}} \\
& { }_{\alpha} \mathbb{S}_{h, \nu}\left(t_{h}^{(1)}\right)=\frac{h^{2} e^{-\frac{h}{\tau^{\nu}}}}{\tau\left(e^{-\frac{h}{\tau^{\nu}}}-\alpha\right)^{2}}
\end{aligned}
$$

Again taking, $u(t)=t_{h}^{(2)}, w(t)=e^{-\frac{t}{\tau^{\nu}}}$ in (6), which gives

$$
\begin{aligned}
\Delta_{\alpha(h)}^{-1}\left[t_{h}^{(2)} e^{\left.-\frac{t}{\tau^{\nu}}\right]}\right. & =t_{h}^{(2)} \Delta_{h}^{-1} e^{-\frac{t}{\tau^{\nu}}}-\Delta_{\alpha(h)}^{-1}\left[\Delta_{\alpha(h)}^{-1} e^{-\frac{1}{\tau^{\nu}}(t+h)} \Delta_{(h)} t_{h}^{(2)}\right] \\
& =t_{h}^{(2)} \frac{h e^{-\frac{t}{\tau^{\nu}}}}{\left(\frac{h}{\tau^{\nu}}-\alpha\right)}-\Delta_{\alpha(h)}^{-1}\left[\frac{h e^{-\frac{1}{\tau^{\nu}}(t+h)}}{h} t_{h}^{(1)}\right] \\
& =t_{h}^{(2)} \frac{\left.h e^{-\frac{h}{\tau^{\nu}}}-\alpha\right)}{\left(e^{\nu}\right.}-\frac{2 h}{\left.\frac{h}{\tau^{\nu}}-\alpha\right)}-\Delta_{\alpha(h)}^{-1}\left[t_{h}^{(1)} e^{-\frac{1}{\tau^{\nu}}(t+h)}-\alpha\right) \\
\Delta_{\alpha(h)}^{-1} t_{h}^{(2)} e^{-\frac{t}{\tau^{\nu}}} & \left.=t_{h}^{(2)} \frac{h e^{-\frac{t}{\tau^{\nu}}}}{\left(e^{-\frac{h}{\tau^{\nu}}}-\alpha\right)}-\frac{\left(e^{-\frac{h}{\tau^{\nu}}}-\alpha\right)\left[t_{h}^{(1)} \frac{h e^{-\frac{1}{\tau^{\nu}}(t+h)}}{h}\left(e^{-\frac{h}{\tau^{\nu}}}-\alpha\right)\right.}{h^{2} e^{-\frac{1}{\tau^{\nu}}(t+2 h)}}\left(e^{-\frac{h}{\tau^{\nu}}}-\alpha\right)^{2}\right] \\
& =t_{h}^{(2)} \frac{h e^{-\frac{t}{\tau^{\nu}}}}{\left(e^{-\frac{h}{\tau^{\nu}}}-\alpha\right)}-\frac{2 h^{2} t_{h}^{(1)} e^{-\frac{1}{\tau^{\nu}}(t+h)}}{\left(e^{-\frac{h}{\tau^{\nu}}}-\alpha\right)^{2}}+\frac{2 h^{3} e^{-\frac{1}{\tau^{\nu}}(t+2 h)}}{h}
\end{aligned}
$$

${ }^{1 *}$ chandrasekarmaths81@gmail.com, ${ }^{2}$ meganathanmath@gmail.com, ${ }^{3}$ vasukishc@gmaflaggend 46 of 152 
ISSN: 2456-8686, Volume 4, Issue 1, 2020:137-152

DOI : http://doi.org/10.26524/cm73

$$
\begin{aligned}
\left.\Delta_{\alpha(h)}^{-1} t_{h}^{(2)} e^{-\frac{t}{\tau^{\nu}}}\right|_{t=0} ^{\infty} & =t_{h}^{(2)} \frac{h e^{-\frac{t}{\tau^{\nu}}}}{\left(e^{-\frac{h}{\tau^{\nu}}}-\alpha\right)}-\frac{2 h^{2} t_{h}^{(1)} e^{-\frac{1}{\tau^{\nu}}(t+h)}}{\left(e^{-\frac{h}{\tau^{\nu}}}-\alpha\right)^{2}}+\left.\frac{2 h^{3} e^{-\frac{1}{\tau^{\nu}}(t+2 h)}}{\left(e^{-\frac{h}{\tau^{\nu}}}-\alpha\right)^{3}}\right|_{t=0} ^{\infty} \\
\left.\Delta_{\alpha(h)}^{-1} t_{h}^{(2)} e^{-\frac{t}{\tau^{\nu}}}\right|_{t=0} ^{\infty} & =-\left.\frac{2 h^{3} e^{-\frac{2 h}{\tau^{\nu}}}}{\left(e^{-\frac{h}{\tau^{\nu}}}-\alpha\right)^{3}} \frac{1}{\tau} \Delta_{\alpha(h)}^{-1} t_{h}^{(2)} e^{-\frac{t}{\tau^{\nu}}}\right|_{t=0} ^{\infty}=-\frac{2 h^{3} e^{-\frac{2 h}{\tau^{\nu}}}}{\tau\left(e^{-\frac{h}{\tau^{\nu}}}-\alpha\right)^{3}} \\
{ }_{\alpha} S_{h, \nu}\left(t_{h}^{(2)}\right) & =-\frac{2 h^{3} e^{-\frac{2 h}{\tau^{\nu}}}}{\tau\left(e^{-\frac{h}{\tau^{\nu}}}-\alpha\right)^{3}} .
\end{aligned}
$$

In general, on induction ' $n$ ' we get (17).

Corollary 3.8 Let $t \in(0, \infty), h>0$ and $\tau>0$, then

$$
{ }_{\alpha} S_{h, \nu}\left(t^{n}\right)=\sum_{r=0}^{n} \frac{(-1)^{n+1} \mathbb{S}_{r}^{n} n ! h^{n+1} e^{\frac{-n h}{\tau^{\nu}}}}{\tau\left(e^{\frac{-h}{\tau^{\nu}}}-\alpha\right)^{n+1}} .
$$

Proof. The proof follows from $(i i)$ of (3), (ii) of (7) and Theorem (3.7)

$$
\begin{aligned}
\Delta_{\alpha(h)}^{-1} t^{n} e^{-\frac{t}{\tau^{\nu}}} & =\Delta_{\alpha(h)}^{-1}\left[\sum_{r=0}^{n} S_{r}^{n} h^{n-r} t_{h}^{(r)}\right] e^{-\frac{t}{\tau^{\nu}}}=\sum_{r=0}^{n} S_{r}^{n} h^{n-r}\left[\Delta_{\alpha(h)}^{-1} t_{h}^{(n)} e^{-\frac{t}{\tau^{\nu}}}\right] \\
& =\sum_{r=0}^{n} S_{r}^{n} h^{n-r}\left[(-1)^{n+1} \frac{h^{n+1} n ! e^{\frac{-n h}{\tau^{\nu}}}}{\tau\left(e^{\frac{-h}{\tau^{\nu}}}-\alpha\right)^{n+1}}\right] \\
{ }_{\alpha} \mathbb{S}_{h, \nu}\left(t^{n}\right) & =\sum_{r=0}^{n} \frac{(-1)^{n+1} \mathbb{S}_{r}^{n} n ! h^{n+1} e^{\frac{-n h}{\tau^{\nu}}}}{\tau\left(e^{\frac{-h}{\tau^{\nu}}}-\alpha\right)^{n+1}} .
\end{aligned}
$$

The following example is the numerical verification of Theorem (3.7).

${ }^{1 *}$ chandrasekarmaths81@gmail.com, ${ }^{2}$ meganathanmath@gmail.com, ${ }^{3}$ vasukishc@gmallagend 47 of 152 
Example 3.9 Taking $n=2$ in Theorem (17), we obtain

$$
{ }_{\alpha} \mathbb{S}_{h, \nu}\left(t_{h}^{(2)}\right)=-\frac{2 h^{3} e^{\frac{-2 h}{\tau^{\nu}}}}{\tau\left(e^{\frac{-h}{\tau^{\nu}}}-\alpha\right)^{3}}=-\frac{h}{\tau} \sum_{r=0}^{\infty} \alpha^{-(r+1)}(r h)_{2}^{(2)} e^{-\frac{r h}{\tau^{\nu}}},
$$

which verified for the values $h=2, \tau=3 \alpha=4$ and $\nu=0.5$ by MATLAB coding given below:

$(16 . * \exp (-2 . * 2 . / 3 . \wedge(0.5))) \cdot /(3 . *(\exp (-2 . / 3 . \wedge(0.5))-4) . \wedge 3)=$ $(2 . / 3) . * \operatorname{symsum}(4 . \wedge(-(r+1)) . * 4 . * r . *(r-1) . * \exp ((-r . * 2) . /(3 . \wedge(0.5))), r, 0$, inf $)$

The following Figure $\mathbf{1}$ is the input function(signal) as polynomial factorial for the time factor $t$ and Figure $\mathbf{2}$ is the fractional generalized Sumudu transform in the frequency domain $\tau$ and also here in the frequency domain the fraction $\nu$ varies as $0.4,0.3,0.2,0.1$ which are generated by MATLAB are shown below.

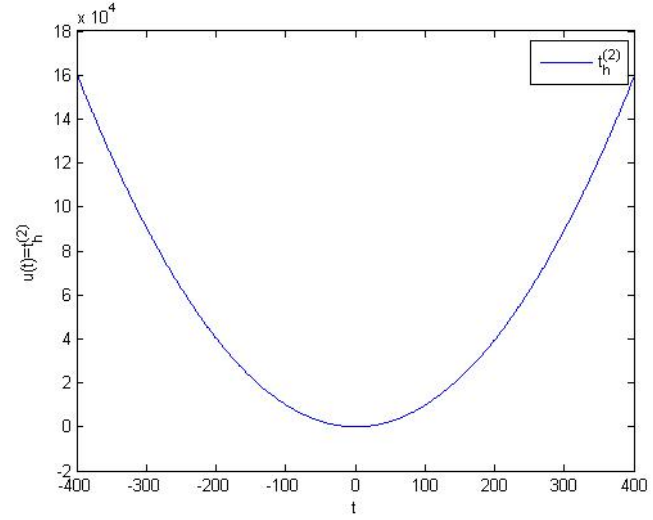

Figure 1: Time(t)

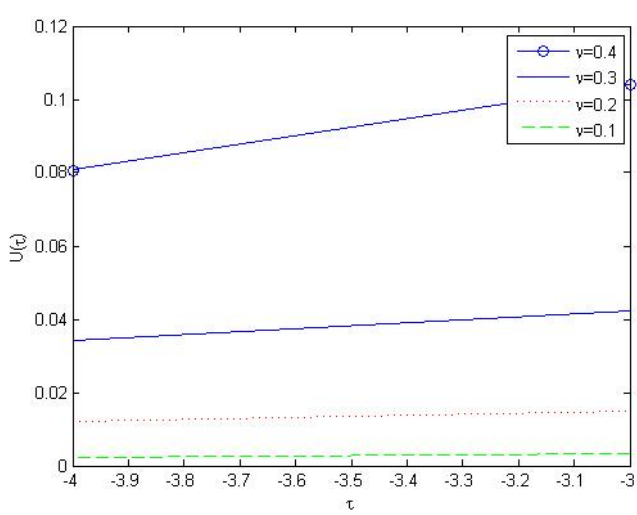

Figure 2: Frequency $(\tau)$

\section{Convolution Product and Fractional Sumudu Transforms}

In this section, we defined convolution product with Fractional Sumudu transforms. The following definitions are motivated using $\alpha(h)$-difference operator.

Definition 4.1 Let $u(t)$ be the real valued function, then the incomplete generalized Sumudu transform is defined by

$$
{ }_{\alpha} \mathbb{S}_{h}[u(t), b]=\left.\frac{1}{\tau} \Delta_{\alpha(h)}^{-1} u(t) e^{-t / \tau^{\nu}}\right|_{0} ^{b}
$$

Definition 4.2 Let $u(t)$ and $v(t)$ are the two real valued functions, then the

${ }^{1 *}$ chandrasekarmaths81@gmail.com, ${ }^{2}$ meganathanmath@gmail.com, ${ }^{3}$ vasukishc@gmalliag@rd 48 of 152 
ISSN: 2456-8686, Volume 4, Issue 1, 2020:137-152

DOI : http://doi.org/10.26524/cm73

convolution product is defined by

$$
(u \circ v)(t)=\left.\Delta_{\alpha(h)}^{-1} u(\xi-t) v(\xi)\right|_{\xi=t} ^{\infty}, \quad t>0
$$

The following lemma shows that the relation between convolution product and fractional Sumudu transform with shift value.

Lemma 4.3 Let $\mu \in \mathbb{R}^{+}, u(t)$ and $v(t)$ are the real valued functions, then

(i) $u \circ e^{-\frac{1}{\mu^{\nu}}}={ }_{\alpha} \mathbb{S}_{h, \nu}[u] \cdot e^{-\frac{1}{\mu^{\nu}}}$

(20), we get $\left(u \circ e^{-\frac{1}{\mu^{\nu}}}\right)(t)=\left.\Delta_{\alpha(h)}^{-1} u(\xi-t) e^{-\frac{1}{\mu^{\nu}} h}\right|_{\xi=t} ^{\infty}$

Taking $t_{1}=\xi-t,\left(u \circ e^{-\frac{1}{\mu^{\nu}}}\right)(t)=\left.\Delta_{\alpha(h)}^{-1} u\left(t_{1}\right) e^{-\frac{1}{\mu^{\nu}}\left(t_{1}+t\right)}\right|_{t_{1}=0} ^{\infty}$

Then, we have

$$
\begin{aligned}
\left(u \circ e^{-\frac{1}{\mu^{\nu}}}\right)(t) & =\left.e^{-\frac{1}{\mu^{\nu}}(t)} \Delta_{\alpha(h)}^{-1} u\left(t_{1}\right) e^{-\frac{1}{\mu^{\nu}}\left(t_{1}\right)}\right|_{t_{1}=0} ^{\infty}, \\
\left(u \circ e^{-\frac{1}{\mu^{\nu}}}\right)(t) & =e^{-\frac{1}{\mu^{\nu}}(t)}{ }_{\alpha} \mathbb{S}_{h, \nu}[u], \\
u \circ e^{-\frac{1}{\mu^{\nu}}} & ={ }_{\alpha} \mathbb{S}_{h, \nu}[u] \cdot e^{-\frac{1}{\mu^{\nu}}(t)} .
\end{aligned}
$$

(ii) Now,

${ }_{\alpha} \mathbb{S}_{h, \nu}[u \circ v]=\left.\Delta_{\alpha(h)}^{-1}(u \circ v)(t) e^{-\frac{1}{\mu^{\nu}}(t)}\right|_{t=0} ^{\infty}=\left.\Delta_{\alpha(h)}^{-1}\left[\left.\Delta_{\alpha(h)}^{-1} u(\xi-t) v(\xi)\right|_{\xi=t} ^{\infty}\right] e^{-\frac{1}{\mu^{\nu}}(t)}\right|_{t=0} ^{\infty}$

Now applying Fubini's Theorem, we get

$$
\begin{aligned}
\left.\Delta_{\alpha(h)}^{-1} e^{-\frac{1}{\mu^{\nu}}(t)}\left[\left.\Delta_{\alpha(h)}^{-1} u(\xi-t) v(\xi)\right|_{\xi=t} ^{\infty}\right]\right|_{t=0} ^{\infty} & =\left.\Delta_{\alpha(h)}^{-1} v(\xi)\left[\left.\Delta_{\alpha(h)}^{-1} u(\xi-t) e^{-\frac{1}{\mu^{\nu}}(t)}\right|_{t=0} ^{\infty}\right]\right|_{\xi=0} ^{\infty} \\
{ }_{\alpha} \mathbb{S}_{h, \nu}[u \circ v] & ={ }_{\alpha} \mathbb{S}_{h, \nu}\left[{ }_{\alpha} S_{h}\left(u\left(t_{1}\right), \xi\right)\right] .
\end{aligned}
$$

The following example is the analysis of the convolution product both numerically and diagrams are generated by MATLAB.

Example 4.4 Consider the following functions

$$
u(t)=\left\{\begin{array}{cc}
e^{-\frac{t}{\tau^{\nu}}}, & t \in(0, \infty) \\
0, & \text { otherwise }
\end{array} \quad, \quad v(t)=\left\{\begin{array}{cc}
t, & t \in(0, \infty) \\
0, & \text { otherwise }
\end{array}\right.\right.
$$

\footnotetext{
${ }^{1 *}$ chandrasekarmaths81@gmail.com, ${ }^{2}$ meganathanmath@gmail.com, ${ }^{3}$ vasukishc@gmalliag@rd 49 of 152
} 
Now we have from (20), we get $(u \circ v)(t)=\left.\Delta_{\alpha(h)}^{-1} e^{-\frac{1}{\tau^{\nu}}(\xi-t)} \xi\right|_{\xi=0} ^{\infty}$. Then using (18), which gives $(u \circ v)(t)=\frac{h^{2} e^{-\frac{1}{\tau^{\nu}}(h-t)}}{\tau\left(e^{-\frac{h}{\tau^{\nu}}}-\alpha\right)^{2}}$. Then the relation as follows

$(u \circ v)(t)=\frac{h}{\tau} \sum_{r=0}^{\infty} \alpha^{-(r+1)}(r h) e^{-\frac{1}{\tau^{\nu}}(r h-t)}=\frac{h^{2} e^{-\frac{1}{\tau^{\nu}}(h-t)}}{\tau\left(e^{-\frac{h}{\tau^{\nu}}}-\alpha\right)^{2}}$

which verified for the values $t=2, h=3, \tau=2, \alpha=2$ and $\nu=0.4$ by (5)

MATLAB coding given bellow:

$(9 . * \exp (-1 . / 2 . \wedge(0.4))) \cdot /(2 . *(\exp (-3 . / 2 . \wedge(0.4))-2) \cdot \wedge(2))=$

$(3 . / 2) . * \operatorname{symsum}(2 . \wedge-(r+1) . * r . * 3 . * \exp (-(3 . * r-2) . /(2 . \wedge(0.4))), r, 0$, inf $)$.

The following Figure 3 explains the input time(t) function $u(t)$ and $v(t)$ and Figure 4 tells that the convolution product of the functions in the frequency $(\tau)$ domain as varying $\nu$ as $0.4,0.5,0.3$ which are generated by MATLAB are shown below.

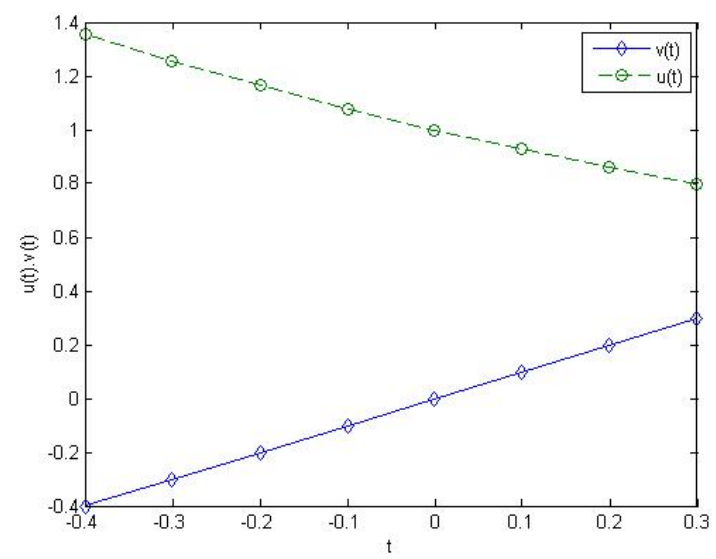

Figure 3: Time(t)

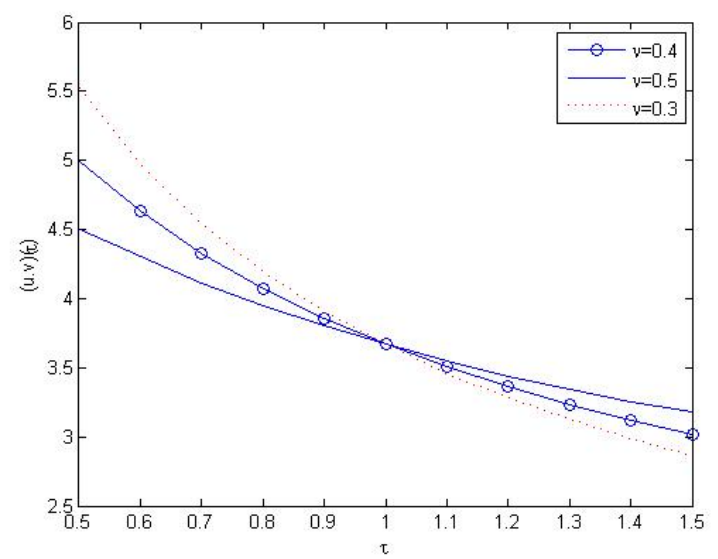

Figure 4: Frequency $(\tau)$

\section{Conclusion}

In this research article, we introduced and derived results on fractional frequency Sumudu transform with shift values and using $\alpha$-difference operator. We believe that this transform is an alternative in the field of difference equations. The more

${ }^{1 *}$ chandrasekarmaths81@gmail.com, ${ }^{2}$ meganathanmath@gmail.com, ${ }^{3}$ vasukishc@gmalliag@r 150 of 152 
ISSN: 2456-8686, Volume 4, Issue 1, 2020:137-152

DOI : http://doi.org/10.26524/cm73

advantage of this research is when $\nu=1, \alpha=1$ and $h \rightarrow 0$, we get the same results on classical Sumudu transform which is existing in the literature.

\section{Acknowledgement}

Catalyzed and financially supported by Tamilnadu State Council for Science and Technology, Dept. of Higher Education, Government of Tamilnadu.

\section{References}

[1] 5. Dumitru Baleanu, Maysaa Alqurashi, Meganathan Murugesan and Britto Antony Xavier Gnanaprakasam, One dimensional fractional frequency Fourier transform by inverse difference operator, Advances in difference equations, 2019-212, https://doi.org/10.1186/s13662-019-2071-y.

[2] Meganathan M, Britto Antony Xavier G and Abdeljawad T, Modeling with fractional Laplace transform by $h$-difference operator, Progress in fractional differentiation and applications(published abstract online).

[3] Atici FM, Eloe PW, A transform method in discrete fractional calculus, International Journal of Difference Equations, 2(2), 2007,165-176.

[4] Butzer PL, Westphal U, An introduction to fractional calculus, World Scientific Press, Singapore, 2000.

[5] Davis PJ, Leonhard Euler Integral Historical Profile of the Gamma function, American Mathematical Society, 66(10), 849-869.

[6] Hamamci SE, An algoritheorem for stabilization of fractional-order time delay systems using fractional-order PID controllers, IEEE Trans. Automat. Control 52 (2007), 1964-1969.

[7] Hilfer R, Applications of Fractional calculus in physics, Word Scientific Press, Singapore, 2000 .

[8] Miller KS, Ross M, Fractional Difference Calculus in Univalent functions, fractional calculus and their applications(Koriyama, 1988), Ellis Horwood Ser. Math. Appl, 1989, 139-152.

[9] Watugala GK, Math J. Educ. Sci. Technol.24(1), 35-43(1993).

[10] MathWatugala GK, Math. Engr.Ind.8(4), 293-302(1998).

[11] Weerakoon S, Math J. Edu. Sci. Technol. 29(4), 618-621(1998).

$\overline{{ }^{1 *} \text { chandrasekarmaths81@gmail.com, }{ }^{2} \text { meganathanmath@gmail.com, }{ }^{3} \text { vasukishc@gmaflaggent } 51 \text { of } 152}$ 
ISSN: 2456-8686, Volume 4, Issue 1, 2020:137-152

DOI : http://doi.org/10.26524/cm73

[12] Weerakoon S, Math J. Educ.Sci.Technol.25(2), 277-283(1994).

[13] Asiru MA, Math J. Educ.Sci. Technol. 32(6), 906-910(2001).

[14] Belgacem FBM, Karubali AA, Appl J.Math. Stoch.Anal.2006, 1-23(2006).

[15] Bohner M, Guseinov Sh, Math J.Appl.365(2010)75-92.

[16] Hilger S, Results Math. 18, 18-56(1990).

[17] Katatbeh QD, Belgacem FBM, Non linear studies 18(1), 1-15(2011).

[18] Kadem A, Baleanu D, Comm. Nonlinear Sc. Numer. Simulat. 2011.01.027 (2011).

[19] Beleanu D, Muslih SI, Rabei EM, Golmankhaneh Alireza K, Golmankhaneh AK, Romanian Reports in Physics 63(1), 3-8(2011).

[20] S. Vasuki, M. Meganathan, B. Chandrasekar and G. Britto Antony Xavier,Modeling with Fractional Frequency Sumudu Transform by Inverse Difference Operator, Online International Interdisciplinary Research Journal, Volume-09, Mar 2019 Special Issue (04).

[21] G. Britto Antony Xavier and M. Meganathan, Analysis of Fractional Alpha Laplace and Extorial Transform Using (h)difference Operator, Journal of Applied Science and Computations, Volume V, Issue XII, December/2018, 2295 2305. - UGC

[22] Meganathan M, T. Abdeljawad, G Britto Antony Xavier and Fahd Jarad, Alpha Fractional Frequency Laplace Transform Through Multiseries, Advance in difference equation (accepted).

${ }^{1 *}$ chandrasekarmaths81@gmail.com, ${ }^{2}$ meganathanmath@gmail.com, ${ }^{3}$ vasukishc@gmallagend 52 of 152 